\title{
Estimating Annotation Cost for Active Learning in a Multi-Annotator Environment
}

\author{
Shilpa Arora, Eric Nyberg and Carolyn P. Rosé \\ Language Technologies Institute \\ Carnegie Mellon University \\ Pittsburgh, PA 15213, USA \\ \{shilpaa, ehn, cprose\}ecs.cmu.edu
}

\begin{abstract}
We present an empirical investigation of the annotation cost estimation task for active learning in a multi-annotator environment. We present our analysis from two perspectives: selecting examples to be presented to the user for annotation; and evaluating selective sampling strategies when actual annotation cost is not available. We present our results on a movie review classification task with rationale annotations. We demonstrate that a combination of instance, annotator and annotation task characteristics are important for developing an accurate estimator, and argue that both correlation coefficient and root mean square error should be used for evaluating annotation cost estimators.
\end{abstract}

\section{Introduction}

Active Learning is the process of selectively querying the user to annotate examples with the goal of minimizing the total annotation cost. Annotation cost has been traditionally measured in terms of the number of examples annotated, but it has been widely acknowledged that different examples may require different annotation effort (Settles et al., 2008; Ringger et al., 2008).

Ideally, we would use actual human annotation cost for evaluating selective sampling strategies, but this will require conducting several user studies, one per strategy on the same dataset. Alternatively, we may be able to simulate the real user by an annotation cost estimator that can then be used to evaluate several selective sampling strategies without having to run a new user study each time. An annotation cost estimator models the characteristics that can differentiate the examples in terms of their annotation time. The characteristics that strongly correlate with the annotation time can be used as a criterion in selective sampling strategies to minimize the total annotation cost.

In some domains, the annotation cost of an example is known or can be calculated exactly before querying the user. For example, in biological experiments it might be calculable from the cost of the equipment and the material used (King et al., 2004). In NLP, sometimes a simplifying assumption is made that the annotation cost for an example can be measured in terms of its length (e.g. seconds of voicemail annotated (Kapoor et al., 2007); number of tokens annotated (Tomanek et al., 2007)). Another assumption is that the number of user annotation actions can be used as a proxy for annotation cost of an example (e.g. number of brackets added for parsing a sentence (Hwa, 2000); number of clicks for correcting named entities (Kristjannson et al., 2004)). While these are important factors in determining the annotation cost, none of them alone can fully substitute for the actual annotation cost. For example, a short sentence with a lot of embedded clauses may be more costly to annotate than a longer sentence with simpler grammatical structure. Similarly, a short sentence with multiple verbs and discontinuous arguments may take more time to annotate with semantic roles than a longer sentence with a single verb and simple subject-verb-object structure (Carreras and Márquez, 2004).

What further complicates the estimation of annotation cost is that even for the same example, annotation cost may vary across annotators (Settles et al., 2008). For example, non-native speakers of English were found to take longer time to annotate part of 
speech tags (Ringger et al., 2008). Often multiple annotators are used for creating an annotated corpus to avoid annotator bias, and we may not know all our annotators beforehand. Annotation cost also depends on the user interface used for annotation (Gweon et al., 2005), and the user interface may change during an annotation task. Thus, we need a general annotation cost estimator that can predict annotation cost for a given annotator and user interface. A general estimator can be built by using annotator and user interface characteristics in addition to the instance characteristics for learning an annotation cost model, and training on data from multiple annotators and multiple user interfaces. Such a general estimator is important for active learning research where the goal is to compare selective sampling strategies independent of the annotator and the user interface.

In this work, we investigate the annotation cost estimation problem for a movie review classification task in a multi-annotator environment with a fixed user interface. We demonstrate that a combination of instance, annotation task and annotator characteristics is important for accurately estimating the annotation cost. In the remainder of the paper, we first present a survey of related work and an analysis of the data collected. We then describe the features used for our supervised learning approach to annotation cost estimation, followed by the experimental setup and results. Finally, we conclude with some future directions we would like to explore.

\section{Related work}

There has been some recent research effort in using supervised learning for estimating annotation cost. The most closely related work is that by Settles et al. (2008) and Ringger et al. (2008). Settles et al. (2008) present a detailed analysis of annotation cost for four NLP applications: named entity recognition, image retrieval, speculative vs. definite distinction, and information extraction. They study the effect of domain, annotator, jitter, order of examples, etc., on the annotation cost.

Results from Settles et al. (2008) are promising but leave much room for improvement. They used only instance level features such as number of entities, length, number of characters, percentage of non-alpha numeric characters, etc. for annotation cost estimation. For three of their tasks, the correlation between the estimated and actual annotation times was in the range ( $R=0.587$ to 0.852$)$. Note that the percentage of variance accounted for by a model is obtained by squaring the $R$ value from the correlation coefficient. Thus, an $R$ value of 0.587 indicates that only about $34 \%\left(R^{2}\right)$ of the variance is accounted for, so the model will make incorrect predictions about ranking in the majority of cases. Nevertheless, we acknowledge that our results are not substantially better, although we argue that this work contributes to the pool of knowledge that will hopefully lead to better performance in the future.

Settles et al. (2008) train and test their estimator on data from the same annotator. Thus, in order to use their model for a new annotator, we would need to first collect data for that annotator and train a model. In our work, a group of annotators annotate the same text, and we train and test on different annotators. We also show that using characteristics of the annotators and annotation task in addition to the instance characteristics improves performance.

Ringger et al. (2008) use linear regression for annotation cost estimation for Part-Of-Speech (POS) tagging. About 30 annotators annotated 36 different instances each. The authors present about 13 descriptive statistics of the data, annotator and annotation task, but in their model they only used number of tokens in the sentence and the number of corrections needed as features. They report that the other variables didn't have a significant effect when evaluated using a Bayesian Information Criterion (from the $\mathrm{R}$ package).

Ringger et al. (2008) noticed that nativeness of the annotator did have an effect on the annotation time, but they chose not to include that feature in their model as they expected to have a similar mix of skills and background in their target annotators. However, if annotation times differ substantially across annotators, then not accounting for this difference will reduce the performance of the model. Also, the low adjusted correlation value for their model $(R=0.181)$ indicates that there is only a weak correlation between the annotation time and a linear combination of the length of the example and the number of corrections. 


\section{Analysis and Experiments}

In this section, we present our annotation methodology and analysis of the data we collected, followed by a description of the features we used.We then present our experimental setup followed by a discussion of our results.

\subsection{Annotation Methodology and Data Analysis}

In this work, we estimate the annotation cost for a movie review classification task. The data we used were collected as part of a graduate course. Twenty annotators (students and instructors) were grouped into five groups of four each. The groups were created such that each group had similar variance in annotator characteristics such as department, educational experience, programming experience, etc. We used the first 200 movie reviews from the dataset provided by Zaidan et al. (2007), with an equal distribution of positive and negative examples. Each group annotated 25 movie reviews randomly selected from the 200 reviews and all annotators in each group annotated all 25 reviews. In addition to voting positive or negative for a review, annotators also annotated rationales (Zaidan et al., 2007), spans of text in the review that support their vote. Rationales can be used to guide the model by identifying the most discriminant features. In related work (Arora and Nyberg, 2009), we ascertain that with rationales the same performance can be achieved with less annotated data. The annotation task with rationales involved a variety of user actions: voting positive or negative, highlighting spans of text and adding rationale annotations. We used the same annotation guidelines as Zaidan et al. (2007). The data has been made available for research purposes ${ }^{1}$. Figure 1 shows a screenshot of the GUI used. We performed an analysis of our data similar to that conducted by Settles et al. (2008). We address the following main questions.

Are the annotation times variable enough? If all examples take a similar time to annotate, then the number of examples can be used as an approximation for the annotation cost. Figure 2 shows the histogram of averaged annotation times (averaged over

\footnotetext{
${ }^{1}$ www.cs.cmu.edu/ shilpaa/datasets/ial/ ial-uee-mr-v0.1.zip
}

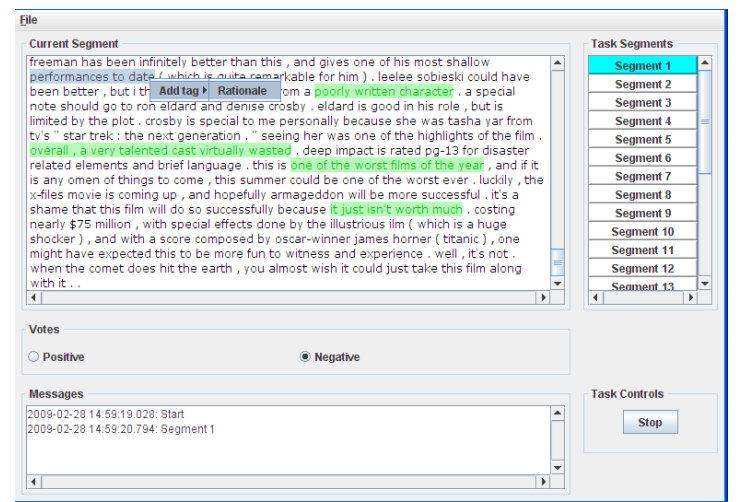

Figure 1: The GUI used for the annotation task. The user selects the review (segment) to annotate from the list in the right panel. The review text is displayed in the left panel. The user votes positive or negative using the radio buttons. Rationales are added by selecting a span of text and right clicking to select the rationale tag. The start/stop button can be used to pause the current task.

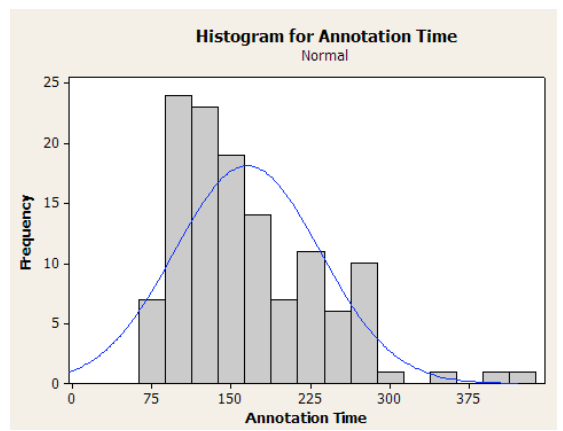

Figure 2: Distribution of averaged annotation times

4 annotators in a group). As can be seen from the mean $(\mu=165 \mathrm{sec}$.) and the standard deviation ( $\sigma=68.85$ ), there is a meaningful variance in the annotation times.

How do the annotation times vary across annotators? A strong correlation between annotation times from different annotators on a set of instances suggests that there are certain characteristics of these instances, independent of the annotator characteristics, that can determine their ranking based on the time it takes to annotate them. We evaluated the pairwise correlation for all pairs of annotators in each group (Table 1). As can be seen, there is significant pairwise correlation in more than half of the pairs of annotators that differ in nativeness (10/16). However, not all such pairs of annotators are associated with significant correlation. This suggests that it is important to consider both instance and annotator characteristics for estimating annotation time. 


\begin{tabular}{|r|r|r|r|}
\hline group & Avg-Na(Std) & Avg-CR(Std) & \#sign-pairs \\
\hline 0 & $2.25(0.96)$ & $0.54(0.27)$ & $4 / 6(4 / 5)$ \\
\hline 1 & $1.75(0.5)$ & $0.45(0.08)$ & $5 / 6(2 / 3)$ \\
\hline 2 & $1(0)$ & $0.13(0.17)$ & $0 / 6(0 / 0)$ \\
\hline 3 & $1.75(0.96)$ & $0.36(0.12)$ & $2 / 6(1 / 5)$ \\
\hline 4 & $2.75(0.5)$ & $0.47(0.04)$ & $6 / 6(3 / 3)$ \\
\hline Avg. & $1.9(0.58)$ & $0.39(0.21)$ & $17 / 30(10 / 16)$ \\
\hline
\end{tabular}

Table 1: The Table shows the average nativeness and average pairwise correlation between annotation times for the members of each group (and their standard deviation). \#sign-pairs shows the fraction of pairwise correlations within the groups that were significant $(p<0.05)$. In brackets, is the fraction of correlations between annotators with different nativeness within the groups that were significant.

The box plot in Figure 3 shows the distribution of annotation times across annotators. As can be seen, some annotators take in general much longer than others, and the distribution of times is very different across annotators. For some, the annotation times vary a lot, but not so much for others. This suggests that using annotator characteristics as features in addition to the instance characteristics may be important for learning a better estimator.

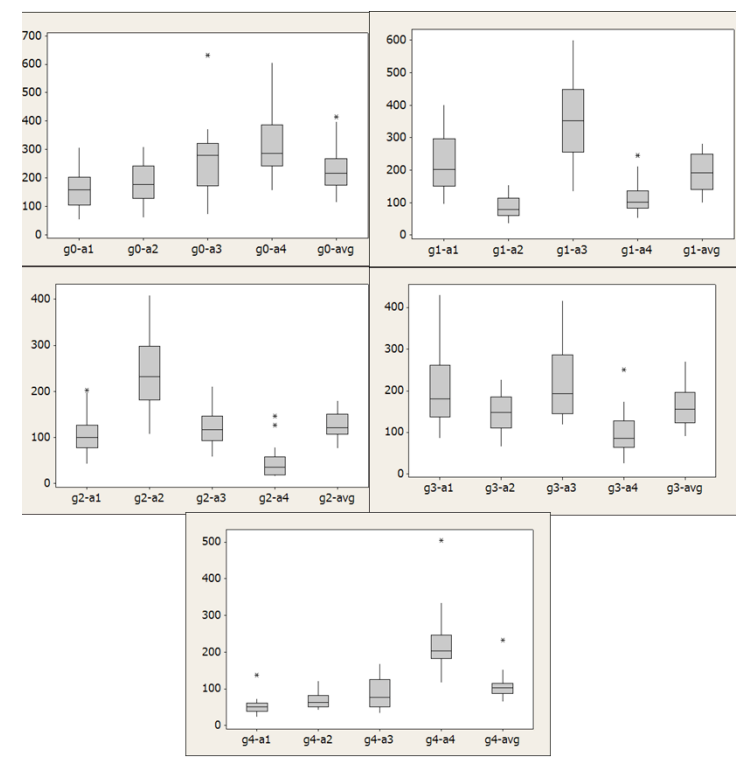

Figure 3: Box plot shows the annotation time (in sec) distribution (y-axis) for an annotator (x-axis) for a set of 25 documents. g0-al represents annotator 1 of group 0 and $g 0-a v g$ represents the average annotation time. A box represents the middle $50 \%$ of annotation times, with the line representing the median. Whiskers on either side span the $1^{\text {st }}$ and $4^{\text {th }}$ quartiles and asterisks indicate the outliers.

\subsection{Feature Design}

We group the features in the following three categories: Instance, Annotation Task and Annotator characteristics.

\subsubsection{Instance characteristics}

Instance characteristics capture the properties of the example the user annotates. Table 2 describes the instance based features we used and the intuition supporting their use for annotation cost estimation. Table 3 shows the mean and standard deviation of each of these characteristics, and as can be seen, these characteristics do vary across examples and hence these features can be beneficial for distinguishing examples.

\subsubsection{Annotation Task characteristics}

Annotation task characteristics are those that can be captured only during or after the annotation task. We used the number of rationales as a feature from this category. In addition to voting for movie reviews as positive or negative, the user also adds rationales that support their vote. More rationales imply more work since the user must look for the relevant span of text and perform the physical action of selecting the span and adding an annotation for each rationale. Table 3 shows the distribution of the average Number of Rationales (NR) per example (averaged over the four annotators for a given set).

\subsubsection{Annotator characteristics}

The annotation cost of an example may vary across annotators. As reported in Table 1, the average correlation for annotators on the same document is low ( $R=0.39$ ) with 17 out of 30 pairwise correlations being significant. Thus, it is important to consider annotator characteristics, such as whether the annotator is a native speaker of English, their education level, reading ability, etc. In this work, we only use nativeness of the annotator as a feature and plan to explore other characteristics in the future. We assigned each annotator a nativeness value. A value of 3 was given to an annotator whose first language is English. A value of 2 was given to an annotator who has a different first language but has either been educated in English or has been in the United States for a long time. A value of 1 was assigned to the remaining annotators. Among the 20 annotators in the study, there were 8 annotators with nativeness value of 1 , and 6 each for nativeness values of 2 and 3 . Table 1 shows the average and standard deviation of the nativeness score in each group. 


\begin{tabular}{|l|l|l|}
\hline Feature & Definition & Intuition \\
\hline $\begin{array}{l}\text { Character } \\
\text { Length (CL) }\end{array}$ & $\begin{array}{l}\text { Length of review in } \\
\text { terms of number of } \\
\text { characters }\end{array}$ & $\begin{array}{l}\text { Longer documents } \\
\text { take longer to anno- } \\
\text { tate }\end{array}$ \\
\hline $\begin{array}{l}\text { Polar word } \\
\text { Count (PC) }\end{array}$ & $\begin{array}{l}\text { Number of words } \\
\text { that are polar (strong } \\
\text { subjective words } \\
\text { from the lexicon } \\
\text { (Wilson et al., 2005)) }\end{array}$ & $\begin{array}{l}\text { More subjectivity } \\
\text { implies user would } \\
\text { need more time to } \\
\text { judge positive vs. } \\
\text { negative }\end{array}$ \\
\hline $\begin{array}{l}\text { Stop word } \\
\text { Percent (SC) }\end{array}$ & $\begin{array}{l}\text { Percentage of words } \\
\text { that are stop words }\end{array}$ & $\begin{array}{l}\text { A high percentage } \\
\text { of stop words im- } \\
\text { plies that the text is } \\
\text { not very complex and } \\
\text { hence easier to read. }\end{array}$ \\
\hline $\begin{array}{l}\text { Avg. Sen- } \\
\text { tence Length } \\
\text { (SL) }\end{array}$ & $\begin{array}{l}\text { Average of the char- } \\
\text { acter length of sen- } \\
\text { tences in the review }\end{array}$ & $\begin{array}{l}\text { Long sentences in a } \\
\text { review may make it } \\
\text { harder to read. }\end{array}$ \\
\hline
\end{tabular}

Table 2: Instance characteristics

\begin{tabular}{|c|r|r|}
\hline Feature & Mean & Standard Deviation \\
\hline CL & 2.25 & 0.92 \\
\hline PC & 41.50 & 20.39 \\
\hline SP & 0.45 & 0.03 \\
\hline SL & 121.90 & 28.72 \\
\hline NR & 4.80 & 2.30 \\
\hline
\end{tabular}

Table 3: Mean and the standard deviation for the feature occurrences in the data.

\subsection{Evaluation Metric}

We use both Root Mean Square (RMS) error and Correlation Coefficient (CRCoef) to evaluate our model, since the two metrics evaluate different aspects of an estimate. RMS is a way to quantify the amount by which an estimator differs from the true value of the quantity being estimated. It tells us how 'off' our estimate is from the truth. CRCoef on the other hand measures the strength and direction of a linear relationship between two random variables. It tells us how well correlated our estimate is with the actual annotation time. Thus, for evaluating how accurate our model is in predicting annotation times, RMS is a more appropriate metric. For evaluating the utility of the estimated annotation cost as a criterion for ranking and selecting examples for user's annotation, CRCoef is a better metric.

\subsection{Experiments \& Results}

We learn an annotation cost estimator using the Linear Regression and SMO Regression (Smola and Scholkopf, 1998) learners from the Weka machine learning toolkit (Witten and Frank, 2005). As men- tioned earlier, we have 5 sets of 25 documents each, and each set was annotated by four annotators. The results reported are averaged over five folds, where each set is one fold, and two algorithms (Linear Regression and SMO Regression). Varying the algorithm helps us find the most predictive feature combinations across different algorithms. Since each set was annotated by different annotators, we never train and test on the data from same annotators. We used the $\mathrm{JMP}^{2}$ and Minitab ${ }^{3}$ statistical tools for our analysis. We used an ANOVA model with Standard Least Squares fitting to compare the different experimental conditions. We make all comparisons in terms of both the CRCoef and the RMS metrics. For significance results reported, we used 2-tailed paired T-test, considering $(p<0.05)$ as significant.

We present our results and analysis in three parts. We first compare the four instance characteristics, annotator and annotation task characteristics; and their combination. We then present an analysis of the interaction between features and annotation time. Finally, we compare the ranking of features based on the two evaluation metrics we used.

\subsubsection{Comparing characteristics for annotation cost estimation}

Instance Characteristics: We compare the four instance characteristics described in Section 3.2.1 and select the most predictive characteristic for further analysis with annotator and annotation task characteristics. As can be seen in Table 4, character length performs the best, and it is significantly better than stop word percent and average sentence length. Character length also outperforms polar word count, but this difference is not significant. Because of the large significant difference between the performance of stop word percent and average sentence length, compared to character length, we do not consider them for further analysis.

Feature Combinations: In Table 5, we compare the feature combinations of instance, annotator and annotation task characteristics. The table also shows the weights for the features used and the constant for the linear regression model trained on all the data. A missing weight for a feature indicates that it wasn't used in that feature combination.

\footnotetext{
${ }^{2}$ http://www.jmp.com/software/

${ }^{3}$ http: //www.minitab.com/
} 


\begin{tabular}{|l|l|l|}
\hline Feature & CR-Coef & RMS \\
\hline CL & $\mathbf{0 . 3 5 8}$ & $\mathbf{1 0 4 . 5 1}$ \\
\hline PC & 0.337 & 105.92 \\
\hline SP & $-0.041^{*}$ & $114.34^{*}$ \\
\hline SL & $0.042^{*}$ & $114.50^{*}$ \\
\hline
\end{tabular}

Table 4: CR-Coef and RMS results for Character Length (CL), Polar word Count (PC), Stop word Percent (SP) and average Sentence Length (SL). Best performance is highlighted in bold. ${ }^{*}$ marks the results significantly worse than the best.

We use only the best performing instance characteristic, the character length. The length of an example has often been substituted for the annotation cost (Kapoor et al., 2007; Tomanek et al., 2007). We show in Table 5 that certain feature combinations significantly outperform character length. The combination of all three features (last row) performs the best for both CRCoef and RMS, and this result is significantly better than the character length (third row). The combination of number of rationales and nativeness (fourth row) also outperforms character length significantly in CRCoef. This suggests that the number of rationales we expect or require in a review and the annotator characteristics are important factors for annotation cost estimation and should be considered in addition to the character length.

\begin{tabular}{|l|l|l|l|l|l|}
\hline CL & NR & AN & Const. & CR-Coef & RMS \\
\hline & & -29.33 & 220.77 & $0.135^{*}$ & $123.93^{*}$ \\
\hline & 17.59 & & 82.81 & 0.486 & 95.29 \\
\hline 0.027 & & & 61.53 & $0.357^{*}$ & $104.51^{*}$ \\
\hline & 19.11 & -40.78 & 153.21 & $0.55^{+}$ & 96.04 \\
\hline 0.028 & & 32.79 & 120.18 & $0.397^{*}$ & $109.85^{*}$ \\
\hline 0.02 & 15.15 & & 17.57 & $0.553^{+}$ & $90.27^{+}$ \\
\hline 0.021 & 16.64 & -41.84 & 88.09 & $\mathbf{0 . 6 2 6}^{+}$ & $\mathbf{8 8 . 4 4}^{+}$ \\
\hline
\end{tabular}

Table 5: CR-Coef and RMS results for seven feature combinations of Character Length (CL), Number of Rationales (NR) and Annotator Nativeness (AN). The values in feature and 'Const.' columns are weights and constant for the linear regression model trained on all the data. The numbers in bold are the results for the best feature combination. ${ }^{*}$ marks the results significantly worse than the best. ${ }^{+}$marks the results significantly better than CL.

The impact of the nativeness feature is somewhat mixed. Adding the nativeness feature always improves the correlation and for RMS, it helps when added to the combined feature (CL+NR) but not otherwise. Although this improvement with addition of the nativeness feature is not significant, it does suggest that annotator characteristics might be important to consider. To investigate this further, we evaluated our assumption that native speakers take less time to annotate. For each set, we compared the average annotation times (averaged over examples) against the nativeness values. For all sets, annotators with nativeness value of 3 always took less time on average than those with nativeness value of 2 or 1 . Between 2 and 1, there were no reliable differences. Sometimes annotators with value of 1 took less time than annotators with value of 2 . Also, for group 2 which had all annotators with nativeness value of 1 , we observed a poor correlation between annotators (Table 1). This suggest two things: 1) our assignment of nativeness value may not be accurate and we need other ways of quantifying nativeness, 2) there are other annotator characteristics we should take into consideration.

\begin{tabular}{|l|l|l|l|l|l|l|}
\hline PC & CL & NR & AN & Const. & CR & RMS \\
\hline & 0.027 & & & 61.53 & $\mathbf{0 . 3 5 8} a b$ & $\mathbf{1 0 4 . 5 x}$ \\
\hline 2.2 & & & & 74.20 & $0.337 \mathrm{a}$ & $105.9 \mathrm{x}$ \\
\hline 0.7 & 0.019 & & & 60.89 & $0.355 \mathrm{~b}$ & $104.9 \mathrm{x}$ \\
\hline \hline & 0.028 & & -32.8 & 120.2 & $\mathbf{0 . 3 9 7} \mathrm{ab}$ & $\mathbf{1 0 9 . 8 x}$ \\
\hline 2.3 & & & -35.5 & 135.1 & $0.382 \mathrm{a}$ & $111.1 \mathrm{x}$ \\
\hline 1.1 & 0.016 & & -34.3 & 121.8 & $0.395 \mathrm{~b}$ & $109.9 \mathrm{x}$ \\
\hline \hline & 0.02 & 15.1 & & 17.57 & $0.553 \mathrm{a}$ & $\mathbf{9 0 . 2 7 x}$ \\
\hline 1.5 & & 15.1 & & 32.02 & $0.542 \mathrm{a}$ & $91.65 \mathrm{x}$ \\
\hline 0.0 & 0.02 & 15.1 & & 17.57 & $\mathbf{0 . 5 5 4 a}$ & $90.40 \mathrm{x}$ \\
\hline \hline & 0.021 & 16.6 & -41.8 & 88.09 & $\mathbf{0 . 6 2 6} \mathrm{a}$ & $\mathbf{8 8 . 4 4 x}$ \\
\hline 1.6 & & 16.5 & -43.5 & 102.8 & $0.614 \mathrm{a}$ & $90.42 \mathrm{y}$ \\
\hline 0.0 & 0.021 & 16.6 & -41.8 & 88.09 & $\mathbf{0 . 6 2 6} \mathrm{a}$ & $88.78 \mathrm{x}$ \\
\hline
\end{tabular}

Table 6: Each block of 3 rows in this table compares the performance of Character Length (CL) and Polar word Count (PC) in combination with Number of Rationales (NR) and Annotator Nativeness (AN) features. The values in feature and 'Const.' columns are weights and constant for the linear regression model trained on all the data. Best performance is highlighted in bold. Results in a block not connected by same letter are significantly different.

Polar word Count and Character Length: As we saw in Table 4, the difference between character length and polar word count is not significant. We further compare these two instance characteristics in the presence of the annotator and annotation task characteristics. Our goal is to ascertain whether character length performs better than polar word count, or vice versa, and whether this difference is significant. We also evaluate whether using both performs better than using any one of them alone. The results presented in Table 6 help us answer these questions. For all feature combinations character length, with and without polar word count, performs 
better than polar word count, but this difference is not significant except in three cases. These results suggests that polar word count can be used as an alternative to character length in annotation cost estimation.

\subsubsection{Interaction between Features and Annotation Time}

As a post-experiment analysis, we studied the interaction between the features we used and annotation time, and the interaction among features themselves. Table 7 reports the pairwise correlation (Pearson, 1895) for these variables, calculated over all 125 reviews. As can be seen, all features have significant correlation with annotation time except stop words percentage and average sentence length.

Note that number of rationales has higher correlation with annotation time $(R=0.529)$ than character length $(R=0.417)$. This suggests that number of rationales may have more influence than character length on annotation time, and a low correlation between number of rationales and character length ( $R=0.238$ ) indicates that it might not be the case that longer documents necessarily contain more rationales. Annotating rationales requires cognitive effort of identifying the right span and manual effort to highlight and add an annotation, and hence more rationales implies more annotation time. We also found some examples in our data where documents with substantially different lengths but same number of rationales took a similar time to annotate. One possible explanation for this observation is user's annotation strategy. If the annotator chooses to skim through the remaining text when enough rationales are found, two examples with same number of rationales but different lengths might take similar time. We plan to investigate the effect of annotator's strategy on annotation time in the future.

A negative correlation of nativeness with annotation time $(R=-0.219)$ is expected, since native speakers $(A N=3)$ are expected to take less annotation time than non-native speakers $(A N=\{2,1\})$, although this correlation is low. A low correlation between number of rationales and nativeness ( $R=0.149)$ suggests that number of rationales a user adds may not be influenced much by their nativeness value. A not significant low correlation ( $R=-0.06)$ between character length and native-

\begin{tabular}{|l|l|l|l|l|l|l|l|}
\hline & AT & CL & NR & AN & PC & SP & SL \\
\hline AT & 1 & & & & & & \\
\hline CL & $\mathbf{0 . 4 2}$ & 1 & & & & & \\
\hline NR & $\mathbf{0 . 5 3}$ & $\mathbf{0 . 2 4}$ & 1 & & & & \\
\hline AN & $\mathbf{- 0 . 2 2}$ & 0.06 & $\mathbf{0 . 1 5}$ & 1 & & & \\
\hline PC & $\mathbf{0 . 4}$ & $\mathbf{0 . 8 9}$ & $\mathbf{0 . 2 8}$ & $\mathbf{0 . 1 1}$ & 1 & & \\
\hline SP & 0.03 & 0.06 & $\mathbf{0 . 1 4}$ & 0.03 & 0.04 & 1 & \\
\hline SL & 0.08 & $\mathbf{0 . 1 5}$ & 0.01 & -0.01 & $\mathbf{0 . 1 4}$ & $\mathbf{- 0 . 1 3}$ & 1 \\
\hline
\end{tabular}

Table 7: Correlation between Character Length (CL), Number of Rationales (NR), Annotator Nativeness (AN), Polar word Count (PC), Stop word Percent (SP), average Sentence Length (SL) and Annotation Time (AT), calculated over all documents (125) and all annotators (20). Significant correlations are highlighted in bold.

ness provides no evidence that reviews with different lengths were distributed non-uniformly across annotators with different nativeness.

The number of polar words in a document has a similar correlation with annotation time as character length $(R=0.4)$. There is also a strong correlation between character length and polar word count $(R=0.89)$. Since reviews are essentially people's opinions, we can expect longer documents to have more polar words. This also explains why there is no significant difference in performance for polar word count and character length (Table 4). A more useful feature may be the information about the number of positive and negative polar words in a review, since a review with both positive and negative opinions can be difficult to classify as positive or negative. We plan to explore these variations of the polar word feature in the future. We also plan to investigate how we can exploit this dependence between characteristics for annotation cost estimation.

\subsubsection{CRCoef Vs. RMS}

We presented our results using correlation coefficient and root mean squared error metrics. Table 8 shows the ranking of the feature combinations from better to worse for both these metrics and as we can see, there is a difference in the order of feature combinations for the two metrics. Also, significance results differ in some cases for the two metrics. These differences suggest that features which correlate well with the annotation times (higher CRCoef rank) can give an accurate ranking of examples based on their annotation cost, but they may not be as accurate in their absolute estimate for simulating annotators and thus might have a lower RMS rank. Thus, it is important to evaluate the user effort esti- 
mator in terms of both these metrics so that the right estimator can be chosen for a given objective.

\begin{tabular}{|l|l|l|}
\hline Rank & CR-Coef & RMS \\
\hline 1 & $(\mathrm{CL}+\mathrm{NR}+\mathrm{AN})$ & $(\mathrm{CL}+\mathrm{NR}+\mathrm{AN})$ \\
\hline 2 & $(\mathrm{CL}+\mathrm{NR})$ & $(\mathrm{CL}+\mathrm{NR})$ \\
\hline 3 & $(\mathrm{NR}+\mathrm{AN})$ & $(\mathrm{NR})$ \\
\hline 4 & $(\mathrm{NR})$ & $(\mathrm{NR}+\mathrm{AN})$ \\
\hline 5 & $(\mathrm{CL}+\mathrm{AN})$ & $(\mathrm{CL})$ \\
\hline 6 & $(\mathrm{CL})$ & $(\mathrm{CL}+\mathrm{AN})$ \\
\hline 7 & $(\mathrm{AN})$ & $(\mathrm{AN})$ \\
\hline
\end{tabular}

Table 8: Ranking of feature combinations.

\section{Towards a General Annotation Cost Estimator}

Our multi-annotator environment allows us to train and test on data from different annotators by using annotator characteristics as features in the annotation cost estimation. A model trained on data from a variety of annotators can be used for recommending examples to annotators not represented in our training data but with similar characteristics. This is important since we may not always know all our annotators before building the model, and training an estimator for each new annotator is costly. Also, in active learning research, the goal is to evaluate selective sampling approaches independently of the annotator. Choosing annotators for supervised annotation cost estimation such that the within group variance in annotator characteristics is high will give us a more generic estimator and a stricter evaluation criterion. Thus, we have a framework that has the potential to be used to build a user-independent annotation cost estimator for a given task.

However, this framework is specific to the User Interface (UI) used. A change in the user interface might require recollecting the data from all the annotators and training a model on the new data. For example, if annotating rationales was made significantly faster in a new UI design, it would have a major impact on annotation cost. An alternative would be to incorporate UI features in our model and train it on several different UIs or modifications of the same UI, which will allow us to use our trained model with a new user interface or modifications of the existing UIs, without having to recollect the data and retrain the model. A few UI features that can be used in our context are: adding a rationale annota- tion, voting positive or negative, etc. The units for expressing these features will be the low-level user interface actions such as number of clicks, mouse drags, etc. For example, in our task, adding a rationale annotation requires one mouse drag and two clicks, and adding a vote requires one click. In a different user interface, adding a rationale annotation might require just one mouse drag.

Using UI features raises a question of whether they can replace the annotation task features; e.g., whether the UI feature for adding rationale annotation can replace the number of rationales feature. Our hypothesis is that number of rationales has more influence on annotation time than just the manual effort of annotating them. It also requires the cognitive effort of finding the rationale, deciding its span, etc. We aim to explore incorporating UI features in our annotation cost estimation model in the future.

\section{Conclusion and Future Work}

In this work we presented a detailed investigation of annotation cost estimation for active learning with multiple annotators. We motivated the task from two perspectives: selecting examples to minimize annotation cost and simulating annotators for evaluating active learning approaches. We defined three categories of features based on instance, annotation task and annotator characteristics. Our results show that using a combination of features from all three categories performs better than any one of them alone. Our analysis was limited to a small dataset. In the future, we plan to collect a larger dataset for this task and explore more features from each feature group.

With the multi-annotator annotation cost estimator proposed, we also motivated the need for a general estimator that can be used with new annotators or user interfaces without having to retrain. We aim to explore this direction in the future by extending our model to incorporate user interface features. We also plan to use the annotation cost model we developed in an active learning experiment.

\section{Acknowledgments}

We would like to thank Hideki Shima for his help with the task setup and Jing Yang for helpful discussions. We would also like to thank all the anonymous reviewers for their helpful comments. 


\section{References}

Shilpa Arora and Eric Nyberg. 2009. Interactive Annotation Learning with Indirect Feature Voting. In Proceedings of NAACL-HLT 2009 (Student Research Workshop).

Xavier Carreras and Lluìs Márquez. 2004. Introduction to the CoNLL-2004 Shared Task: Semantic Role Labeling. http://www.1si.upc.edu/ rslconll/st04/st04.html.

Gahgene Gweon, Carolyn Penstein Ros'e, Joerg Wittwer and Matthias Nueckles. 2005. Supporting Efficient and Reliable Content Analysis Using Automatic Text Processing Technology. In proceedings of INTERACT 2005: 1112-1115.

Robbie A. Haertel, Kevin D. Seppi, Eric K. Ringger and Janes L. Cattoll. 2008. Return on Investment for Active Learning. In proceedings of NIPS Workshop on Cost Sensitive Learning.

Rebecca Hwa. 2000. Sample Selection for Statistical Grammar Induction. In proceedings of joint SIGDAT conference on Empirical Methods in NLP and Very Large Corpora.

Ashish Kapoor, Eric Horvitz and Sumit Basu. 2007. Selective supervision:Guiding supervised learning with decision-theoretic active learning. In proceedings of IJCAI, pages 877-882.

Ross D. King, Kenneth E. Whelan, Ffion M. Jones, Philip G. K. Reiser, Christopher H. Bryant, Stephen H. Muggleton, Douglas B. Kell and Stephen G. Oliver. 2004. Functional Genomics hypothesis generation and experimentation by a robot scientist. In proceedings of Nature, 427(6971):247-52.

Trausti Kristjansson, Aron Culotta, Paul Viola and Andrew Mccallum. 2004. Interactive Information Extraction with Constrained Conditional Random Fields. In proceedings of AAAI.

Karl Pearson. 1895. Correlation Coefficient. Royal Society Proceedings, 58, 214.

Eric Ringger, Marc Carmen, Robbie Haertel, Kevin Seppi, Deryle Lonsdale, Peter McClanahan, Janes L. Cattoll and Noel Ellison. 2008. Assessing the Costs of Machine-Assisted Corpus Annotation through a User Study. In proceedings of LREC.

Burr Settles, Mark Craven and Lewis Friedland. 2008. Active Learning with Real Annotation Costs. In proceedings of NIPS Workshop on Cost Sensitive Learning.

Alex J. Smola and Bernhard Scholkopf 1998. A Tutorial on Support Vector Regression. NeuroCOLT2 Technical Report Series - NC2-TR-1998-030.

Katrin Tomanek, Joachim Wermter and Udo Hahn. 2007. An approach to text corpus construction which cuts annotation costs and maintains reusability of annotated data. In proceedings of EMNLP-CoNLL, pp. 486495.

Theresa Wilson, Janyce Wiebe and Paul Hoffmann. 2005. Recognizing Contextual Polarity in PhraseLevel Sentiment Analysis. In proceedings of HLT/EMNLP, Vancouver, Canada.

Ian H. Witten and Eibe Frank. 2005. Data Mining: Practical machine learning tools and techniques. 2nd Edition, Morgan Kaufmann, San Francisco.

Omar Zaidan, Jason Eisner and Christine Piatko. 2007. Using "annotator rationales" to improve machine learning for text categorization. In Proceedings of NAACL-HLT, pp. 260-267, Rochester, NY. 